\section{RefWorks investigated - an appropriate bibliographic management solution for health students at King's College London?}

\section{Andria McGrath}

\begin{abstract}
Author
Andria McGrath is an Information Specialist at King's College London, involved in training and liaison in the Biomedical School. She also specialises in bibliographic software.
\end{abstract}

\section{Email: andria.mcgrath@kcl.ac.uk}

\begin{abstract}
A small scale pilot project was set up to investigate whether the introduction of web-based bibliographic software at King's College London would prove useful to undergraduate and taught postgraduate students, and to establish the support and training requirements. Customisation of the interface and extensive local preparation aimed to make the service as easy to use as possible, and to test whether it could be used successfully with the minimum of extra training. The service was primarily introduced to a cohort of biomedical students. A questionnaire directed at all ${ }^{\circ}$ registered users revealed a high degree of satisfaction with the service. Some would have liked specific training, but many were happy to receive support in a variety of different ways.
\end{abstract}

\section{Introduction}

King's has for several years provided site licence access to the bibliographic software programs from Thomson ResearchSoft. Reference Manager and EndNote are used extensively by academic staff and research students. They are also available on the public access workstations and could be used by undergraduate or taught postgraduate students. However these programs are quite complex and require some effort to master. Training sessions of two and a half to three hours are provided by ISS to introduce the basics.

Not only would it be difficult to offer this amount of training to undergraduates and taught postgraduates, but it was felt that few of the students would be prepared to devote this amount of time to learning to use the software. In addition, although the software was available on the public access computers, students could not use it outside of College unless they invested in personal copies of the software.

During 2004 staff in the Information Services \& Systems (ISS) Research and Learning Liaison team investigated two web-based bibliographic management programs, WriteNote from Thomson Research Soft, and RefWorks from CSA, to decide whether either of these would be useful at King's. The advantage of web-based software for students is that it is relatively simple to use and, because it is web based, it is available from any networked computer at no cost to the individual, the licence being paid centrally. After experimenting using the trial access provided, the team concluded that RefWorks offered the more convenient interface and options for interacting with online databases.

\section{Methodology}

We decided to offer a pilot RefWorks service from September 2004 to gauge the level of interest and to determine the support implications of providing this software. The program was made available to all, but was only actively promoted during the pilot period to a cohort of students within the School of 
Biomedical Sciences. These students included $2^{\text {nd }}$ and $3^{\text {rd }}$ year undergraduates, many of whom would be required to write long essays including citations and reference lists as part of their course modules, and taught postgraduate students who would all have to write research project dissertations.

\section{Decision to use Athens authentication}

Every effort was made to make the service as easy to use as possible. We first had to decide whether to use Athens authentication. In the UK, RefWorks can be accessed either by Athens authentication, on and off campus, or by ip address authentication on campus, and by a group code plus individual login name and password off campus. IP authentication had some attraction for ease of use, however we felt that the group code login off-campus could be a bit confusing. There is an advantage in using the group code method, in that it enables users to share databases by sharing the login details. This is not possible with Athens authentication as login details are for one person only and cannot be shared.

After seeking clarification from RefWorks and consultation with some other UK RefWorks users we decided to choose Athens as the default access method. Our users are familiar with using Athens on and off campus. This would be the simplest off-campus method, and off-campus use was an important aspect of the choice of this online software. The other method was not ruled out, as the welcome email sent to users registering with RefWorks after logging in with Athens, can be customised by the institution, and this was written to include details of the other method, but stressing that Athens was the normal route.

\section{Preparation}

RefWorks allows customisation of the web interface by the subscribing institution, so, work was put in to ensure that those accessing the service with a King's Athens account were presented with the appropriate import filters for the main online bibliographic databases used at
King's. King's RefWorks web pages were also created to reflect the appropriate online databases

(http://www.kcl.ac.uk/iss/ir/manage/bibsoft/refw $\mathrm{ks}$ ), and a printed user guide was written using appropriate examples (http://www.kcl.ac.uk/ content/1/c4/43/02/refworks.pdf).

This user guide was road tested on three groups of ISS staff (including most of the ISS help desk staff) in short 'training' sessions. In these sessions the service was not demonstrated or taught by the 'trainer', rather, the staff were given a copy of the userguide to see whether it was clear enough to enable them to use the software without actual training. By watching and listening to the comments and problems experienced by these 'guinea pigs' the user guide was progressively improved to provide clearer instructions. The inclusion of ISS helpdesk staff in this exercise ensured that they were also ready to answer any queries brought to them during the pilot.

\section{Promotion of the pilot service}

As we were concerned about the level of training support that might be required, a major aim of the pilot was to determine whether the provision of a basic user guide and supporting web pages might be sufficient to enable most users to use the software effectively.

Consequently the service was introduced to the pilot students in just a five minute demonstration as part of their normal online database searching training sessions in October 2004. They were provided with a copy of the user guide, which also highlights the King's RefWorks support web pages, and were given the option to contact the tutor if they had any problems using the program. This brief demonstration was shown to 292 students in total, at 16 training sessions. The service was promoted in a minor way by some other members of the Liaison team during the pilot year, where they felt it was appropriate for particular students or groups. However the user guide was not made generally available, and there was no general publicity for the service. 


\section{Questionnaire}

A questionnaire was seen to be a simple data collection technique for the purposes of this small scale project, with a view to pursuing a larger cohort and more sophisticated data collection strategies at a later stage, if the service was adopted and more widely promoted. An online questionnaire directed at those who had registered to use the service hopefully would prompt a high response rate, would be inexpensive and gather sufficient data to provide a snap shot of opinion. However, it would not of course elicit any comments from those who had not decided to register after being shown the software in action.

The online questionnaire was devised to test whether the support provided for RefWorks had been sufficient to enable students to use the program effectively, and to gauge their satisfaction with the software and the support materials. RefWorks provides an email list of all those who have self registered to use the service. In mid April 2005 emails were sent to those on this list (312) inviting them to fill in the online questionnaire.

It was known that some people would have registered either during the training sessions, or having heard about RefWorks in some other way, but would not subsequently have used the program in earnest. We wanted to hear from some of these people to find out why they had not decided to use RefWorks, as well as from those who had used the program. The email made this clear, and also offered a $£ 30$ book token prize draw for all those completing the questionnaire and providing their contact details.

\section{Statistics from the RefWorks site}

Institutional RefWorks administrators can access statistics on the use of the program by registered users. These statistics were also examined to give additional understanding of the use of RefWorks at King's.

\section{Questionnaire results}

312 emails were sent and there were 74 responses, a response rate of $23 \%$.

The largest group of respondents was from the School of Biomedical Sciences (42\%), as would be expected, since it was to these students that the program was specifically introduced. A significant proportion was from the School of Medicine (16\%) and other health schools (19\%), with $23 \%$ from the non-health Schools in the College, and ISS staff.

\section{Question I - How did you find out about RefWorks?}

Some respondents included more than one way in which they had found out about the program.

- 44 (59\%) found out about RefWorks from ISS staff, at a presentation, a database training session, or by personal or email contact.

- 10 were informed by academic staff

- 2 had used the program outside King's.

- 29 (39\%) discovered the program for themselves mainly by finding the King's RefWorks web pages (24)

\section{Question 2: Have you used RefWorks - if not, why not?}

Forty-five respondents had used RefWorks and 29 had not. The reasons respondents gave for not using the service are summarised in Figure 1. 


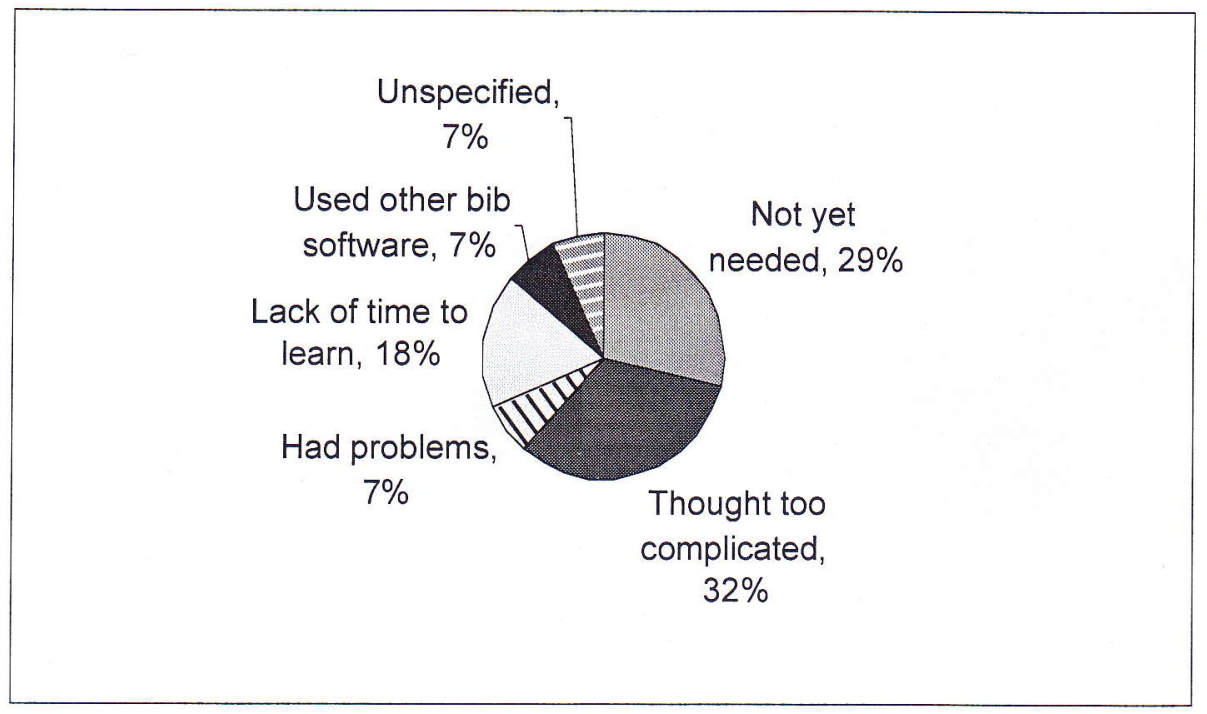

\section{Figure 1: Reasons for not using RefWorks (29 respondents)}

Of the 29 who had not used the program, 15 filled in some or all of the remainder of the questionnaire although they were not required to do so. Fourteen respondents did not give any more responses, and therefore for the remaining results the total number of possible respondents was each question was 60 .

\section{Question 3 -The King's RefWorks userguide}

Twenty-seven respondents had seen the userguide

- $14(52 \%)$ had found the userguide a useful introduction,

- $3(11 \%)$ had not found it useful

- $10(37 \%)$ did not express an opinion.

\section{Question 4 -The King's RefWorks website}

25 respondents had used the website $(42 \%)$, and of these:

- $18(72 \%)$ found it gave them sufficient information to use RefWorks effectively

- $3(12 \%)$ did not find that it gave them enough information

- $4(16 \%)$ did not respond.

\section{Question 5 - support \& training requirements}

$57 \%$ of possible respondents said they would have attended a one-hour training session if it had been offered, $38 \%$ would not and $5 \%$ did not respond.

Twelve respondents had requested help with using RefWorks;

- 6 at an ISS help desk

- 2 by emailing ISS Enquiry services

- 3 in other ways

When asked their preferred method of obtaining support, 26 responded and the results are shown in Figure 2. 


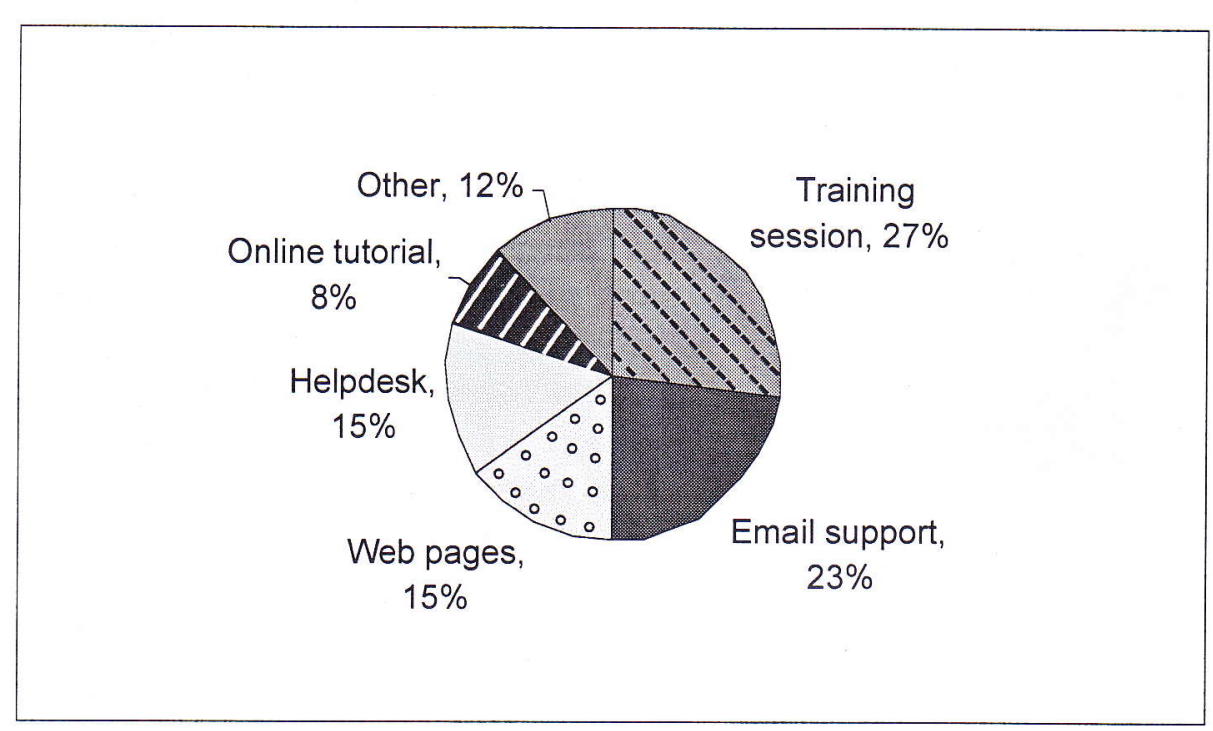

Figure 2: Preferred method for obtaining support (26 respondents)

\section{Question 6 - methods used to populate the RefWorks database}

Respondents were asked to indicate all the methods they had used, and which they had used most.

- The most popular method was direct export from online databases, which was used by 31 $(52 \%)$ respondents, and was used more than other methods by 25 of these.

- Another very popular method (30 respondents, 50\%) was direct connection searching, 16 using it to search PubMed and 8 to search library catalogues. Nine said they used this method most.

- Manual entry was used by 28 respondents, . and for 6 of these it was used most.

- Sixteen used the method of saving a text file and then importing, and 4 used this method most.

This question was included to determine whether users had been able to use the different methods of populating their RefWorks database with the help of the King's user guide, web pages and/or help provided within the program. Direct export was the only method demonstrated when the software was shown to the pilot group, and is the most straightforward, but it was encouraging that many of the respondents had been able to use the other methods.

\section{Question 7 - Use of Write-N-Cite}

Write-N-Cite is the RefWorks MS Word plug-in, which enables the creation of a Word document including citations and a reference list, drawn from records in the user's RefWorks database, and formatted in a specified reference style.

Thirty one respondents (52\%) had used Write$\mathrm{N}$-Cite and 29 had not. Results of the question asking how easy or otherwise they found it are shown in Figure 3.

$71 \%$ of users found it very easy or easy to use, and none found it 'very difficult.' 


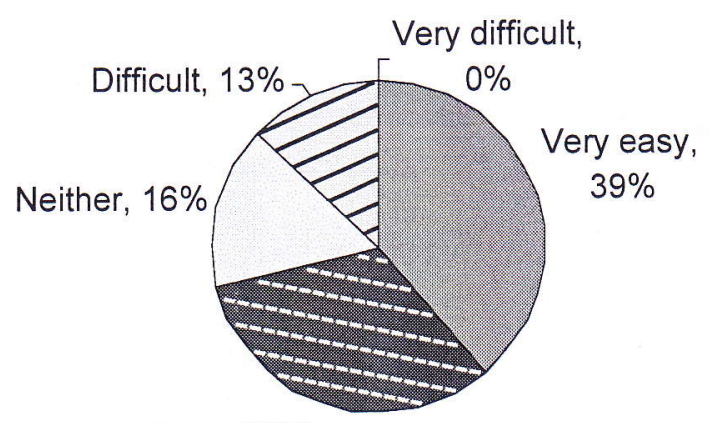

Easy, $32 \%$

Figure 3: Cite-N-Write Ease of use (31 respondents)

Question 8 - satisfaction with the program Would you recommend RefWorks to a friend

Results are shown in Figure 4

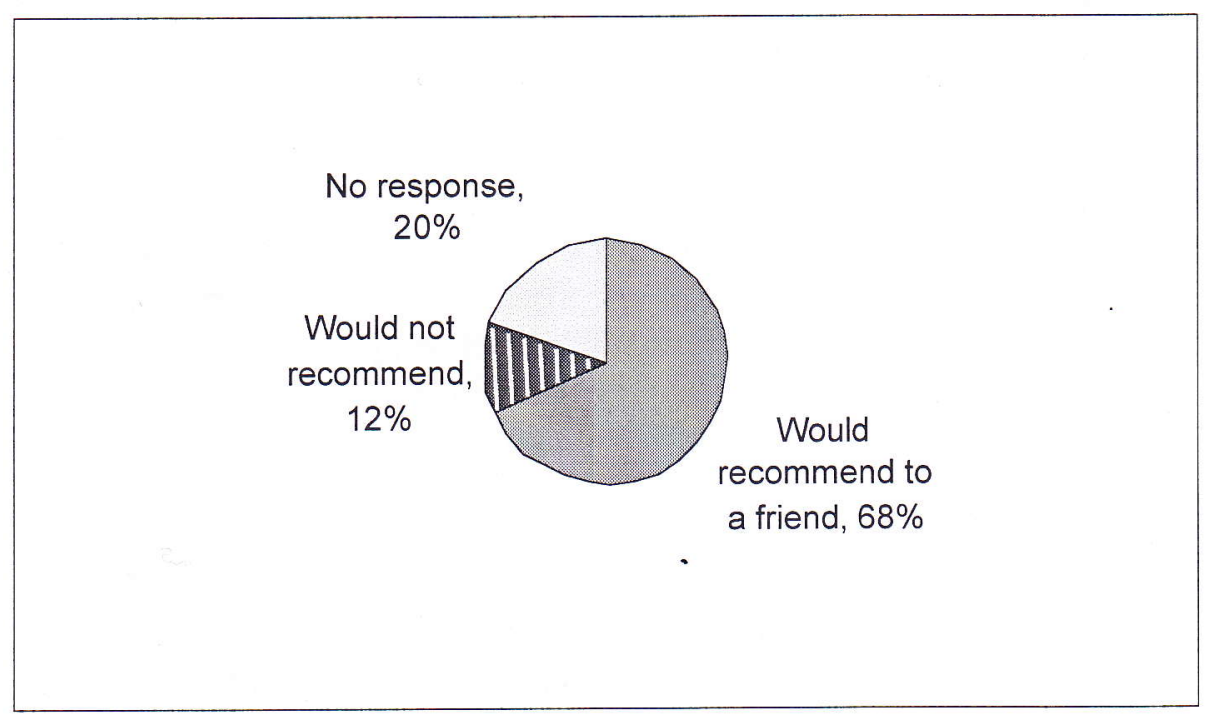

Figure 4 - Would you recommend RefWorks to a friend? (60 possible respondents)

Thirty two responses were given for what was liked best about the program, some respondents giving more than one. The results are shown in Figure 5. 


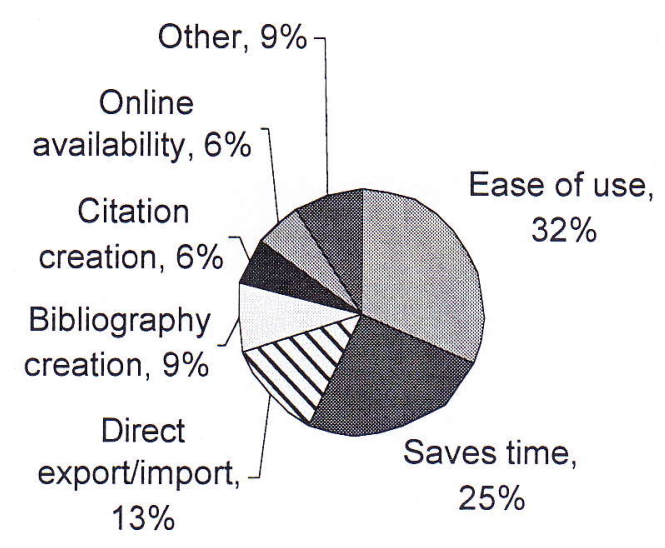

Figure 5: What was liked best about RefWorks (32 responses)

\section{What was disliked about the program?}

A variety of dislikes were mentioned. There were 22 comments

- 5 mentioned Cite-N-Write problems

- 8 found it confusing or difficult

- 2 found not being able to import references from certain databases a problem

- 2 found it time consuming.

\section{Other comments on the program}

Four users were particularly impressed by the program, one commenting "Can't imagine having done my dissertation without it!"

\section{Statistics available from the RefWorks site}

The questionnaire results revealed that 45 respondents had used RefWorks to some extent. To obtain an estimate of how many other registered users of the program might be actively using it, the statistics provided for RefWorks institutional administrators were examined, including the number of log-ins and the number of references added to each person's RefWorks database. The total number of registered users on July 52005 was 349. A large proportion of these users had only logged in once or twice; however, 87 people had added more than 5 refs and logged in more than twice, so these might be considered to be using the program seriously. Considering that the service had only been briefly demonstrated to about 300 students, and not otherwise actively promoted this seemed to be a reasonable number of users.

\section{Conclusions}

The number of students to which this pilot service was promoted and the number of questionnaire responses, particularly for some questions not answered by all respondents, was not sufficient to allow the results to be submitted to rigorous statistical analysis. However, within the numbers responding, the feedback was very largely positive in terms of satisfaction with the software and the support provided. In particular the $68 \%$ of 60 possible respondents who would have recommended the software to a friend was felt to be a sufficient endorsement for continuing to subscribe to RefWorks at King's and it was decided that in the subsequent academic year the service would be promoted to all undergraduate and taught postgraduate students.

In the light of the evidence, however limited in scope, concerning ease of use and support issues, we decided to introduce the software, as in the pilot, by including a demonstration as part of existing database training. However fiftyseven percent of respondents did indicate that 
they would have attended a training session if it had been offered, and therefore additional training will also be provided in the form of sign-up sessions at lunch times or after $5.00 \mathrm{pm}$. The take up of this training provision and the use of help desk and other enquiry support will be monitored.

The ISS Liaison team at King's has welcomed the provision of this software, which should prove a valuable extra opportunity to reinforce good citation habits in students, in cooperation with academic staff. It should help students to understand the techniques and importance of good referencing, and avoid plagiarism due to lack of awareness. The provision of one or more King's-approved reference styles on the King's RefWorks interface is being considered. We plan to engage with academic staff concerning this new service and actively promote it to them, so that they are fully aware of its availability and usefulness.

The current research methodology was flawed in that it restricted us to a limited and biased population by only questioning registered users. Further research is required in order to investigate the opinions and attitudes of those students who do not register to use the service, in order to identify the barriers to uptake and use. The next phase of the research project will identify a larger cohort of students from a number of disciplines and pursue a range of additional collection strategies, including interviews and focus groups, pre- and post training in the use of the resource. Observing a cohort over time will enable us to measure the impact that the web-based bibliographic management resource has upon their general information literacy skills. The challenge will be to capture the processes and changes in their approach to information management as they advance through their studies. 